\title{
Trendige Superfoods: Placebo oder Wundermittel?
}

\author{
Gabriele Leitner
}

\begin{abstract}
Der Begriff „Superfood“ war schon Anfang des 20. Jahrhunderts bekannt, in der Umgangssprache ist er aber erst seit Kurzem populär. Das Europäische Informationszentrum für Lebensmittel (EUFIC) bezeichnet als Superfood „Lebensmittel, insbesondere Obst und Gemüse, die aufgrund ihres Nährstoffgehaltes einen höheren gesundheitlichen Nutzen als andere Nahrungsmittel aufweisen“" 1$]$.
\end{abstract}

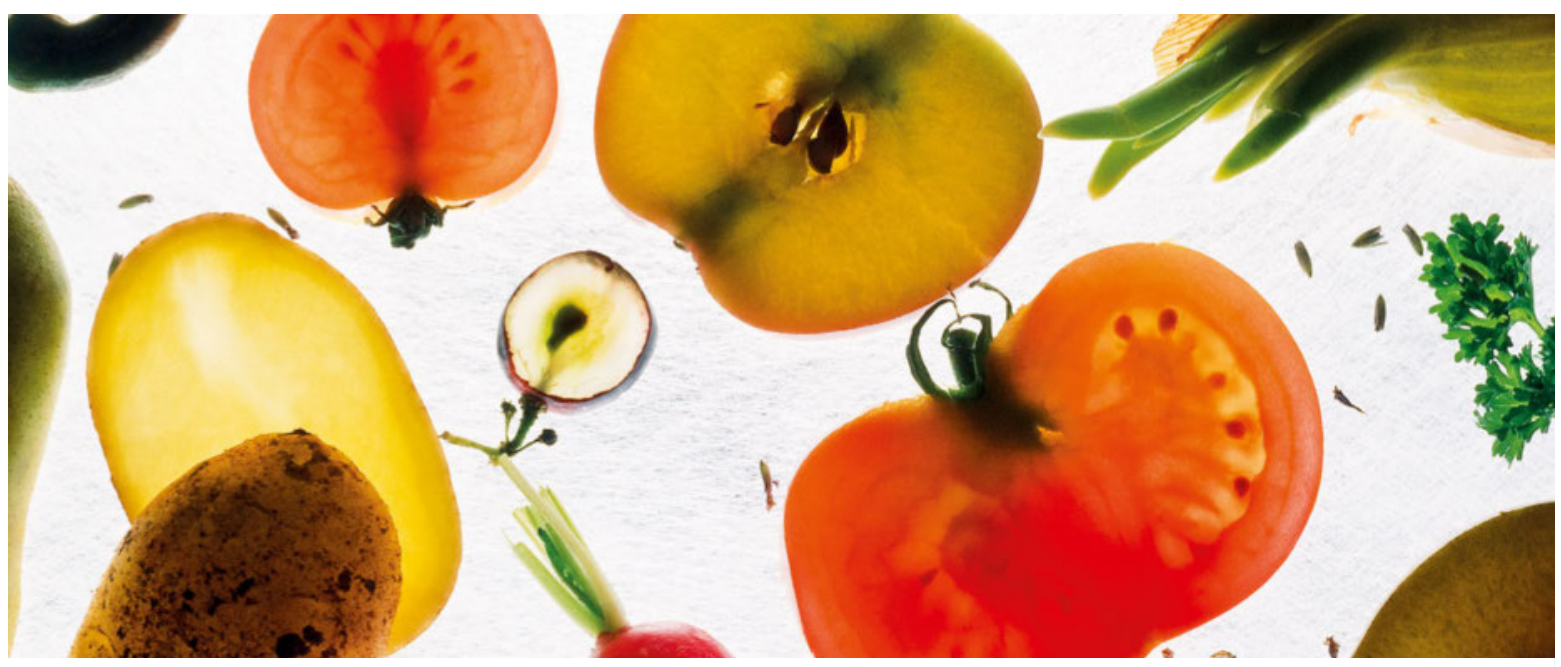

Warum in die Ferne schweifen? Heimisches Gemüse, Obst, Nüsse und Ölsaaten warten mit genauso vielen positiven Inhaltsstoffen auf wie die „trendigen“ Exoten. @ ccvision

\section{Superfood - was steckt dahinter?}

Als vermeintliche Leistungselixiere, die zusätzlich schlank und attraktiv machen, liegen Superfoods gerade voll im Trend. Im Wesentlichen handelt es sich bei Superfood um einen gerne genutzten Marketingbegriff, der vor allem dazu dient, den Produkten ein positives Image zu verleihen. Eine offizielle Definition oder gesetzliche Regelung gibt es jedoch nicht.

Meist handelt es sich bei Superfoods um pflanzliche Lebensmittel wie Früchte, Gemüse, Samen, Kerne und Nüsse, ergänzt durch Algenarten wie Spirulina, AFA und Chlorella. Angeboten werden sie in verschiedenen Formen: als ganze Frucht, als Säfte, in Pulverform, gemahlen oder als Extrakt, in Form von Ölen, Körnern oder Samen.

Die Anwendung zielt vor allem darauf ab, andere Speisen wie Müslis, Backwaren, Smoothies, Salate, Suppen und
Desserts aufzuwerten, oder es handelt sich um spezifische Tees. Häufig werden sie auch als Nahrungsergänzungsmittel in Kapsel- oder Pulverform angeboten [2].

Der Hype um Superfoods zeigt sich aber nicht nur beim Einkaufen, sondern auch in den Medien. Eine einfache Internet-Suche nach dem Wort Superfood ergibt Millionen von Treffern. Sie stammen vor allem aus Gesundheits- und Ernährungsblogs, Online-Magazinen und Anbietern von Nahrungsergänzungsmitteln. Zahlreiche Rezeptportale ergänzen diese mit vielfältigen Ideen rund um die Anwendung und Zubereitung.

Hersteller und überzeugte Fans werben für nahezu jedes Superfood, regelmäßigen Genuss vorausgesetzt, mit einem breiten Spektrum an Gesundheitsversprechen. Nicht selten wird diesen Lebensmitteln auch eine heilsame Wirkung nachgesagt. 


\section{WERBUNG MIT DER GESUNDHEIT}

Angepriesen wird bei Superfoods vor allem die antioxidative Wirkung der Inhaltsstoffe als Wunderwaffe gegen freie Radikale. Diese entstehen durch Umwelteinflüsse, wie z. B. UV-Strahlung oder Abgase, und schädigen das Zellgewebe. Antioxidanzien tragen dazu bei, freie Radikale unschädlich zu machen.

\section{Nachhaltigkeit, Transport und Verarbeitungsgrad}

Vielfach handelt es sich bei Superfoods um Produkte aus exotischen Ländern wie Chia-Samen, Acai-Beere, Camu Camu, Goji-Beere, Granatapfel, Kokosnuss oder Avocado. Bevor sie in unseren Regalen landen, haben sie schon lange Transportwege zurückgelegt.

\section{Merke}

Frisch sind Superfoods meist reich an wertvollen Inhaltsstoffen. Allerdings sind sie frisch bei uns fast nicht zu bekommen.

Die Produkte werden oft unreif geerntet, stark verarbeitet, lagern wochenlang auf Schiffen und werden häufig durch Konservierungsmittel haltbar und damit transportfähig gemacht. Schwefeldioxid bietet Schutz vor Schädlingen und Schimmel, außerdem erhält es die Farbe. Benzoesäure (E211) und Kaliumsorbat (E202) verhelfen Säften zu längerer Haltbarkeit. Der Zusatz von Ascorbinsäure (E 302) trägt dazu bei, Oxidationsprozesse zu verhindern. Darüber hinaus hat bei der Verarbeitung und Haltbarmachung die Temperatur einen großen Einfluss auf die Inhaltsstoffe. Der Gehalt an sekundären Pflanzenstoffen wie Polyphenolen und Flavonoiden ist höher, wenn Gefriertrocknung (unter Vakuum und bei niedrigen Temperaturen) statt Lufttrocknung angewendet wird. Vitamin $C$ reagiert ebenfalls empfindlich auf Lager- und Trocknungsvorgänge bei höheren Temperaturen. Ähnliches gilt auch für Vitamin E, wenn auch in geringerem Ausmaß [3]. Wie viele von den beworbenen, wertvollen Inhaltsstoffen unter den beschriebenen Voraussetzungen noch beim Verbraucher ankommen, ist fraglich.

Werden Extrakte hergestellt, müssen oft wichtige Komponenten der Pflanze entfernt werden. Vor allem antioxidativ-wirkende Substanzen können dadurch verändert oder abgebaut werden oder verlieren möglicherweise ihre Wirksamkeit.

Der stark wachsende Markt erfordert in den Herkunftsländern eine zunehmende Professionalisierung. Wälder werden abgeholzt, Pflanzenschutzmittel, Dünger und andere Chemikalien eingesetzt, um der Nachfrage in den Industrieländern gerecht zu werden [4]. Der weltweite
Transport ist im Steigen. Eine Mehrbelastung der Umwelt und eine hohe Treibhausemission sind die Folge. Der gesundheitliche Benefit der exotischen Produkte ist daher aus diesen ökologischen Gründen kritisch zu hinterfragen.

\section{Exoten versus heimische Alternativen}

Je exotischer das Lebensmittel, desto undurchsichtiger ist für Verbraucher, ob sie überhaupt das richtige Produkt vor sich haben. Die steigende Nachfrage nach einem bestimmten Produkt kann mit den vorhandenen Kapazitäten oft nicht befriedigt werden. Das führt dazu, dass es zu immer mehr Verwechslungen oder gar Fälschungen kommt.

\section{Ein stets wachsender Markt stellt angesichts der Gefahr von Verwechslungen selbst für Experten eine immer größere Herausforderung dar.}

Eine Alternative für die exotischen Superfoods sind die einheimischen Superfoods. Hierzu zählt zum Beispiel die Preiselbeere. Sie lässt sich sehr gut mit den aus China stammenden Goji-Beeren vergleichen: gleiche Nährstoffe und ähnlicher Vitamin- und Mineralstoffgehalt. Auch Kohl, Brokkoli, Brennnesseln, Aronia-Beeren, Hanfsamen, Kürbiskerne, Walnüsse, Sanddorn und Weizengras haben einiges zu bieten.

Nicht außer Acht zu lassen ist der heimische Apfel. Er liefert viele Vitamine und sekundäre Pflanzenstoffe und hat damit auch eine „gesundheitsfördernde Wirkung“ zu bieten. Wie heißt es doch so schön: „An apple a day keeps the doctor away!“

\section{Superfoods - was sagt die Wissenschaft?}

Um die Wahrheit vom Hype zu unterscheiden, ist es wichtig, die wissenschaftlichen Beweise hinter den Superfood-Behauptungen der Medien genau zu hinterfragen. Für viele der bioaktiven Substanzen gibt es zwar erste Untersuchungen, doch reichen diese nicht aus, um eine Empfehlung abzugeben. Meist stammen die Ergebnisse aus Tier- oder Zellversuchen und können nicht auf Anwendungen am Menschen übertragen werden.

Humanstudien existieren in einem nur sehr geringen Ausmaß und sind meist auf ein kleines Probandenkollektiv beschränkt. Zudem wurden sie in den meisten Fällen ohne Kontrollgruppe und mit sehr hohen Dosierungen durchgeführt. Diese hohen Dosierungen sind im Rahmen einer normalen Ernährung in der Regel nicht realistisch und daher nicht erreichbar. Darüber hinaus sind die physiologischen Wirkungen vieler dieser Nahrungsmittel nicht von langer Dauer. Dies bedeutet, dass sie häufig konsu- 
miert werden müssen, um ihre gesundheitlichen Vorteile nutzen zu können.

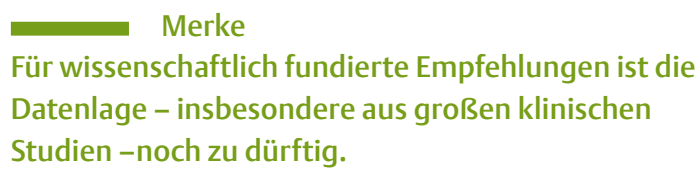

Angesichts der Tatsache, dass Menschen normalerweise Kombinationen von Nahrungsmitteln zu sich nehmen, spiegelt das Auswählen eines einzigen Inhaltsstoffes nicht den tatsächlichen Konsum wider. Auch ist nicht auszuschließen, dass der gleichzeitige Verzehr einer breiteren Auswahl von Nahrungsmitteln die Nährstoffaufnahme verbessert. Beta-Carotin in Karotten und Spinat wird beispielsweise leichter aufgenommen, wenn es zusammen mit einer Fettquelle wie Salatdressing gegessen wird. Dies lässt erkennen, dass eine Ernährung, die auf einer Vielzahl von nahrhaften Lebensmitteln basiert, im Gegensatz zu einer Diät mit nur einer Handvoll Superfoods einen deutlichen Vorteil bietet.

Dazu kommt, dass einzelne Superfoods als Extrakt in Form von Nahrungsergänzungsmitteln angeboten werden. Die Dosierungen des Inhaltsstoffes unterliegen jedoch keiner gesetzlichen Regelung. Sie unterscheiden sich je nach Hersteller, weshalb sich auch die Wirkung zum Teil erheblich unterscheiden kann.

\section{Beispiele für Superfoods}

Heidelbeeren oder Blaubeeren zählen zu den beliebtesten Superfoods und sind daher auch am besten untersucht. Die hohen Konzentrationen an antioxidativ wirksamen Pflanzeninhaltsstoffen, den Anthocyanen, zeigten in Studien krebshemmende Wirkung [6]. Im Versuch mit Ratten ließ sich durch das antioxidative Potenzial der Blaubeeren der alterungsbedingte Gedächtnisverlust positiv beeinflussen und zum Teil sogar umkehren [7].

Granatapfelsaft soll den Blutdruck senken [8] und zur Reduzierung von oxidativem Stress beitragen [9] - beides signifikante Risikofaktoren für Herz-Kreislauf-Erkrankungen. Auch Rote-Beete-Saft wird als herzgesundes Superfood propagiert. Das in beträchtlichen Mengen darin enthaltene Nitrat wird vom Körper in Stickstoffmonoxid umgewandelt, was den Blutdruck senkt und die Blutgerinnung beeinflusst [10].

Cranberries und Aronia-Beeren verfügen ebenfalls über hohes antioxidatives Potenzial und halten Herz und Gefäße gesund. Cranberries werden zudem zur Vorbeugung von Blasenentzündungen empfohlen. Diese gesundheitsbezogenen Aussagen wurden von der Europäischen Behörde für Lebensmittelsicherheit (EFSA) jedoch mit „nicht ausreichend wissenschaftlich bewiesen “ beurteilt und dürfen daher nicht beworben werden [11].
Chia-Samen sind zwar eine gute Quelle für mehrfach ungesättigte Fettsäuren und Proteine, zeigen aber keine eindeutigen Vorteile gegenüber dem heimischen Leinsamen. Ihr Nährstoffgehalt und ihre Wirkung auf die Verdauung sind identisch, nicht aber der Preis: Chia-Samen kosten das 5- bis 10-Fache des Leinsamens.

\section{ORAC-WERTE}

Die antioxidative Kapazität eines Nahrungsmittels wird häufig mit dem ORAC-Wert (Oxygen Radical Absorbance Capacity) charakterisiert. Dieser besagt, wie viele freie Radikale mit einem Gramm Saft oder Frucht neutralisiert werden können. „Die ORAC-Werte sind jedoch reine Laborwerte. Die bei der Messung ablaufende Reaktion findet so im menschlichen Körper nicht statt.“ Deshalb sagt der ORAC-Wert eines Lebensmittels oder eines Nahrungsbestandteils nichts über dessen gesundheitsfördernde und antioxidative Wirkung im menschlichen Organismus aus. Werbung mit dem ORAC-Wert ist verboten, da es sich um eine nicht zugelassene nährwertbezogene Angabe gemäß der Health-Claims-Verordnung handelt [11].

\section{Fazit}

Die Idee von Lebensmitteln mit außergewöhnlichen gesundheitlichen Vorteilen ist attraktiv und hat das öffentliche Interesse voll und ganz erreicht. Die Wissenschaft hat in diesem Bereich gezeigt, dass bestimmte Inhaltsstoffe von Lebensmitteln und Getränken eine positive Wirkung auf die Gesundheit zeigen. Dies spiegelt sich auch in der Existenz anerkannter gesundheitsbezogener Angaben wider, für die die Europäische Behörde für Lebensmittelsicherheit die wissenschaftliche Evidenzbasis als ausreichend überzeugend einschätzt [11]. Gleichzeitig ist es aber unrealistisch, von einer breiten Palette an „Superfoods “ zu erwarten, dass sie unser Wohlbefinden entscheidend verbessern.

\section{KERNAUSSAGEN}

Auch wenn sie keine wahren Wunder vollbringen, liefern die meisten Vertreter der Superfood-Liste eine Vielzahl an Nährstoffen und leisten damit einen wertvollen Beitrag zu einer gesunden und bedarfsdeckenden Ernährung. Ein gesundheitlicher Mehrwert exotischer Superfoods im Vergleich zu heimischen Produkten ist jedoch nicht gegeben. Ihr besonderer Wert liegt eher darin, individuelle Vorlieben zu befriedigen und dadurch die erhoffte Wirkung, ähnlich wie bei einem Placebo, tatsächlich eintreten zu lassen. 


\section{Interessenkonflikt}

Die Autorin gibt an, dass kein Interessenkonflikt besteht.

\section{Autorinnen/Autoren}

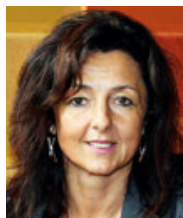

\section{Gabriele Leitner}

Ernährungswissenschaftlerin und Diätologin Dr. rer. nat. Gabriele Leitner ist seit 2009 FH-Dozentin und Modulverantwortliche am Studiengang Diätologie der FH St. Pölten. Ihre Schwerpunkte in der Lehre sind: Ernährungslehre, Allergologie, Rheumatologie, Immunologie, Neurologie und alternative Kostformen.

\section{Korrespondenzadresse}

FH-Prof. Mag. Dr. Gabriele Leitner

Fachhochschule St. Pölten

Department Gesundheit

Matthias Corvinus-Straße 15

3100 St. Pölten

E-Mail: gabriele.leitner@fhstp.ac.at

\section{Literatur}

[1] European Food Information Council, 2016: Superfood: Was verbirgt sich wirklich dahinter? Im Internet: www.eufic.org/ en/healthy-living/article/the-science-behind-superfoods/; Stand: 27.03 .2018

[2] Haller D, Grune T, Rimbach G. Biofunktionalität der Lebensmittelinhaltsstoffe. Berlin Heidelberg: Springer; 2012

[3] Caballero B, Finglas P, Toldrá F. Encyclopedia of Food and Health. München: Elsevier Science; 2015

[4] Koerber K, Männle T, Leitzmann C. Vollwert-Ernährung. Konzeption einer zeitgemäßen und nachhaltigen Ernährung. 11. Aufl. Stuttgart: Haug; 2012
[5] Kowalla J. Klimaschutz und Ernährung, München, GRIN Verlag; 2010. Im Internet: www.grin.com/document/176301/; Stand: 28.03 .2018

[6] Yi W, Fischer J, Krewer G, Akoh CC. Phenolic compounds from blueberries can inhibit colon cancer cell proliferation and induce apoptosis. Agric Food Chem 2005; 53 : $7320-7329$

[7] Malin DH, Lee DR, Goyarzu P et al. Short-term blueberry-enriched diet prevents and reverses object recognition memory loss in aging rats. Nutrition 2011; 27: 338-342

[8] Lynn A, Hamadeh $\mathrm{H}$, Leung WC et al. Effects of pomegranate juice supplementation on pulse wave velocity and blood pressure in healthy young and middle-aged men and women. Plant Foods Hum Nutr 2012; 67: 309-314

[9] Aviram M, Dornfeld L, Rosenblat M et al. Pomegranate juice consumption reduces oxidative stress, atherogenic modifications to LDL, and platelet aggregation: studies in humans and in atherosclerotic apolipoprotein E-deficient mice. Am J Clin Nutr 2000; 71: 1062-1076

[10] Webb AJ, Patel N, Loukogeorgakis S et al. Acute blood pressure lowering, vasoprotective, and antiplatelet properties of dietary nitrate via bioconversion to nitrite. Hypertension 2008; 51: 784-790

[11] EFSA. EU Register of nutriton and health claims made on foods. Im Internet: http://ec.europa.eu/nuhclaims/; Stand: 18.03.2018

[12] Delgado-Lista J, Perez-Martínez P, Lopez-Miranda J, Perez-jimenez F. Long chain omega-3 fatty acids and cardiovascular disease: a systematic review. Br J Nutr 2012; 107 (Suppl 2): S201-213

[13] Goldberg RJ, Katz J. A meta-analysis of the analgesic effects of omega- 3 polyunsaturated fatty acid supplementation for inflammatory joint pain. Pain 2007; 129: 210-223

\section{Bibliografie}

DOI https://doi.org/10.1055/a-0549-9206

Ernährung \& Medizin 2018; 33: 55-58

(c) Georg Thieme Verlag KG Stuttgart · New York ISSN 1439-1635 